\title{
Decreasing Microtubule Actin Cross-Linking Factor I Inhibits Melanoma Metastasis by Decreasing Epithelial to Mesenchymal Transition
}

This article was published in the following Dove Press journal: Cancer Management and Research

\author{
Xiaoying Wang' \\ Xiao Jian' \\ Jun Dou (iD) ${ }^{2}$ \\ Zicheng $\mathrm{Wei}^{3}$ \\ Fengshu Zhao (D) ${ }^{2}$ \\ 'Wuxi School of Medicine, Jiangnan \\ University, Wuxi, People's Republic of \\ China; ${ }^{2}$ Department of Pathogenic \\ Biology and Immunology, School of \\ Medicine, Southeast University, Nanjing, \\ People's Republic of China; ${ }^{3}$ Department \\ of Stomatology Affiliated Hospital of \\ Jiangnan University, Wuxi, People's \\ Republic of China
}

Correspondence: Jun Dou; Fengshu Zhao Department of Pathogenic Biology and Immunology, Medical School, Southeast University, 87 Dingjiaqiao Road, Nanjing 210009, People's Republic of China Tel +86-25-83272454; +86-25-83272506 Email njdoujun@seu.edu.cn; Fengshu_zhao@I26.com
Background: The microtubule actin cross-linking factor 1 (MACF1) is involved in cellular migration, adhesion, and invasion processes. Its abnormal expression initiates tumor cell proliferation and metastasis in numerous cancer types.

Methods: In this study, we utilized short hair-pin RNA interference of MACF1 to assess the inhibitory effects on the metastatic potential of B16F10 melanoma cells both in vitro and in vivo a mouse model.

Results: The MACF1 expression was increased in B16F10 cells-induced tumor tissues; while the down-regulation of MACF1 impacted the B16F10 melanoma cell metastatic behavior by decreasing the ability of colony formation and invasion in vitro as well as inhibiting B16F10 cells-induced tumor growth and lung metastasis in vivo. The results of Western blot and immunohistochemistry indicated that the expression of E-cadherin and Smad-7 was significantly increased whereas the expression of N-cadherin and TGF- $\beta 1$ was significantly decreased in tumor tissue of mice challenged with the B16F10/MACF1-RNAi cells when compared with the B16F10 cells challenged mice.

Conclusion: The data presented in this study demonstrated that down-regulated MACF1 expression decreased B16F10 melanoma metastasis in mice by inhibiting the epithelial to mesenchymal transition program. Thus, MACF1 may be a novel target for melanoma therapy.

Keywords: melanoma, microtubule actin cross-linking factor 1, metastasis, epithelial to mesenchymal transition

\section{Introduction}

Melanoma, the deadliest type of skin cancer, is one of the most invasive and metastatic cancers with an increasing incidence and a very poor prognosis worldwide. Both the outgrowth of primary and metastatic melanoma pose significant and unprecedented clinical challenges. ${ }^{1,2}$ Although surgical ablation is the most efficient treatment prior to melanoma invasion and the formation of metastases, a significant percentage of patients remains refractory. As a result, the five-year survival of patients is low, and malignant melanoma will become a formidable disease once cancer cells colonize other tissues. ${ }^{3,4}$ Immunotherapy with immune checkpoint inhibitors (inhibitory monoclonal antibodies against the cytotoxic T-lymphocyte antigen-4 (CTLA-4) and programmed death 1 (PD-1/ PDL1) and targeted therapy (Vemurafenib and nivolumab) acted as a certain efficiency on metastatic melanoma; unfortunately, these immune-based therapies are not fully responsive in some advanced stage melanoma. ${ }^{5,6}$ In this regard, it is desirable to adopt 
an efficient therapeutic strategy to overcome the shortage in successful treatment of metastatic melanoma based on immune checkpoint inhibitors and targeted therapy.

Emerging evidence has accumulated describing the molecular function of microtubule actin cross-linking factor 1 (MACF1), belonging to the plakin family of proteins, ${ }^{7,8}$ which plays an essential role in various cellular processes. MACF1 is also involved in metastatic invasion in which organization of the cytoskeleton is a critical contributing element to tumor progression in various human cancers. ${ }^{9,10}$ In a recent report, it was shown that MACF1 was predominately present in grade III-IV astrocytomas and grade IV glioblastoma, but not in normal brain tissue and astrocytes or lower grade brain tumors. ${ }^{10}$ These findings suggested that MACF1 function may not only be related to the regulation of normal cellular physiology, including cytoskeleton organization, but also to cellular pathology, such as tumor progression in numerous cancers.

In the present study, we hypothesized that MACF1 down-regulation might inhibit melanoma metastasis in B16F10 cells-induced melanoma mice by decreasing epithelial to mesenchymal transition (EMT) of B16F10 melanoma cells. Our findings showed that B16F10 cell invasion and colony formation as well as oncogenicity and lung metastasis were markedly decreased both in vitro and in vivo after knocking down MACF1 expression in B16F10 cells. Our data indicated that the expression of E-cadherin and Smad-7 was significantly enhanced whereas the expression of $\mathrm{N}$-cadherin and TGF- $\beta 1$ was significantly decreased in mice that were challenged with B16F10/MACF1-RNAi cell, thereby indicating inhibition of B16F10 cell EMT in vivo. Our current study demonstrated that MACF1 in melanoma may serve as a potential therapeutic target to enhance the efficacy of treatment of melanoma.

\section{Materials and Methods Cell Line}

The B16F10 murine melanoma cell line is syngeneic in C57BL/6 mice, and was ordered from the Cellular Institute of China (Shanghai, China). Cells were cultured at $37^{\circ} \mathrm{C}$ and $5 \% \mathrm{CO}_{2}$ in RPMI 1640 medium that was supplemented with $10 \%$ fetal bovine serum (FBS) containing $100 \mathrm{Uml}^{-1}$ penicillin $\mathrm{G}$ sodium and $100 \mathrm{mg} \mathrm{mL}^{-1}$ streptomycin sulfate.

\section{Mice}

C57BL/6 mice at six weeks of age and weighing 17$18 \mathrm{~g}$ were purchased from the Yangzhou University
(Yangzhou, China). Mice were housed under pathogenfree conditions. Experiments were performed in compliance with the guidelines of the Animal Research Ethics Board of Jiangnan University (Jiangnan, China).

\section{Immunoblot Analysis}

Samples for immunoblot analysis $\left(1 \times 10^{6}\right.$ different cells cultured for $48 \mathrm{~h}$ ) were collected using CytoBuster protein extraction buffer (Novagen, Germany) according to the manufacturer's guidelines. Immunoblot was performed proteins were separated on $12 \%$ SDS-polyacrylamide gel, and were used for Western blot analysis using a WesternBreeze Kit (Invitrogen, Carlsbad, CA, USA). Briefly, proteins (10 $\mu \mathrm{g}$ / lane) were transferred onto a Polyvinylidene Fluoride (PVDF) Film, and membranes were blocked with $4 \%$ dry milk in Trisbuffered saline with Tween-20 (TBST) for $1 \mathrm{~h}$ at $20^{\circ} \mathrm{C}$. Membranes were then incubated overnight at $4{ }^{\circ} \mathrm{C}$ with rabbit anti-mouse/human MACF1 ((A-3): sc-377532, Santa Cruz Biotechnology, USA), rabbit anti-mouse/human E-cadherin (CST, 24E10, 1: 500), Smad7(ab216428,1:500), TGF- $\beta 1$ (Novus,7F6,1:1000), and N-cadherin (CST, D4R1H,1: 500), respectively. Membranes were rinsed 3 times for 5 min with an antibody wash solution and incubated with goat anti-rabbit fluorescence secondary antibody. The immunoreactive bands were visualized by an Odyssey scanning instrument (LI-COR Odyssey Imaging System, USA). ${ }^{11,12}$

\section{Construction of Vector Containing siRNAs Targeting the MACFI Encoding Gene}

The Small interfering RNA was synthesized by Hanheng Biotechnology Co. Ltd (Shanghai, China). The primer sequences were as follows: siRNA-MACF1\# forward, 5'-GG C AGGUGAAGCUAGUGAAdTdT-3'; reverse, 5'-UUCAC UAG CUUCACCUGC CdTdT-3'; siRNA-MACF2\# forward, 5'-GAGUACAAGUGC AGAGUAAdTdT-3';reverse,5'-UU ACUCUGCACUUGUACUCdTdT-3'; siRNA-MACF3\# forward,5'-CGAUCUUGAUGUCAGAUAAdTdT-3'; reverse, 5' -UUAUCUGACAUCA AGAUCGdTdT-3'; siRNA-negative forward, 5'-UUCU CCGAACGUGUCACGUdTd-3'; reverse, 5'-ACGUGACAC GUUCGGAGAA dTdT-3'. Primers were synthesized by Gene and Technology of China (Shanghai, China). ${ }^{6}$

\section{Cell Proliferation}

B16F10/MACF1-RNAi cells and B16F10 cells were respectively seeded at a density of $5 \times 10^{3}$ cells/well into 96-well plates and incubated in a $\mathrm{CO}_{2}$ incubator for $0,24,48$, and 
$72 \mathrm{hrs}$ at $37^{\circ} \mathrm{C}$. Subsequently, cells were incubated for an additional $4 \mathrm{~h}$ with $10 \mu \mathrm{L}$ of CCK-8 Cell Proliferation and Cytotoxicity Assay Kit. Absorbance values were determined at a wave length of $450 \mathrm{~nm}$ by a microplate reader (Bio-Rad, USA).

\section{Colony Formation in Soft Agar Media}

The colony formation ability of B16F10 cells transfected with pSUPER-EGFP1-MACF1-shRNA1 or a scrambled control siRNA was investigated. Colony with a diameter of more than $75 \mu \mathrm{m}$ or with more than 50 cells was counted as 1 positive colony based on our previous reports. ${ }^{13,14}$ One hundred single B16F10 cells were resuspended in $0.8 \mathrm{~mL}$ growth media, containing $0.3 \%$ low melting temperature agarose (Promega, Madison, WI, USA), and plated in triplicate in a 24-well plate over a base layer of $0.8 \mathrm{~mL}$ growth media containing $0.6 \%$ low melting temperature agarose. Plates were incubated for 12-14 days until colonies were formed. The clone formation efficiency was calculated as (number of colonies/number of cells inoculated) $\times 100 \%$.

\section{BI6FI0 Cell Invasion Assay}

The invasion ability of $\mathrm{B} 16 \mathrm{~F} 10$ cells transfected with MACF1-RNAi or the control scrambled RNAi was evaluated using a transwell invasion assay as previously described. ${ }^{15,16}$ Briefly, transwell inserts with $8 \mu \mathrm{m}$ pores were coated with Matrigel (20 $\mu \mathrm{g} /$ well; Becton Dickinson, Waltham, MA, USA). Then, $1 \times 10^{5}$ single-cell suspension in serum-free RPMI-1640 medium were respectively seeded in the upper chamber in RPMI 1640 medium, supplemented with $10 \% \mathrm{FBS}$. After incubation at $37^{\circ} \mathrm{C}$, cells that invaded to the lower surface of the Matrigelcoated membranes were fixed with $70 \%$ ethanol and stained with trypan blue. The cells from five randomly selected fields were counted under a light microscope.

\section{In vivo Animal Experiments}

Eighteen C57BL/6 mice (females, 5 and 6 weeks, and weighting 16-18g) were randomly divided into three groups (six mice per group): a B16F10 cell group, a B16F10-scrambled-RNAi (B16F10- negative) group, and a B16F10/MACF1-RNAi group. Mice were subcutaneously (s.c.) injected in the back with $2 \times 10^{5}$ B16F10 cells, $2 \times 10^{5}$ B16F10-negative cells, and $2 \times 10^{5}$ B16F10/MACF1-RNAi cells, respectively. For the lung metastatic experiment, 18 mice were randomly divided into three groups as mentioned above, and each mouse was intravenously injected with $2 \times 10^{5}$ B16F10 cells, $2 \times 10^{5}$ B16F10negative cells, and $2 \times 10^{5}$ B16F10/MACF1-RNAi cells, respectively. Except for the daily observation of mouse general conditions, such as overall behavior, feeding, body weight, and appearance of fur, tumor formation was monitored every three days, and 2-dimensional measurements of individual tumors were performed from each mouse. In the lung metastatic experiment, mice were euthanized 21 days after injection of cells. Experiments were performed in duplicate. ${ }^{17,18}$

\section{Lung Metastases Nodes}

To evaluate the inhibitory effect on lung tumor metastases, B16F10 cell induced tumor mice were executed and lung tissues were removed. Subsequently, metastatic tumor nodes were counted with a tenfold megaloscope uwing a naked eye. ${ }^{19}$

\section{Immunohistochemistry}

After mice were euthanized, B16F10 cells-forming tumors were removed for immunohistochemical and immunoblot analysis. Immunostaining was performed as reported previously. ${ }^{17,20}$ Briefly, $4 \mu \mathrm{m}$-thin formalin fixed and paraffinembedded tumor sections were incubated overnight at $4{ }^{\circ} \mathrm{C}$ using rabbit anti-mouse/human E-cadherin, N-cadherin, Smad7, and TGF- $\beta 1$ antibodies, respectively. Sections were washed with PBS and incubated with horseradish peroxidase (HRP)-conjugated streptavidin (Invitrogen), and a chromogenic reaction was performed by using a Liquid DAB Substrate Pack according to the manufacturer's guideline. Stained cells were counted in 10 random and nonoverlapping fields at a high magnification of $\times 400$.

\section{Statistical Analysis}

Data were presented as the mean plus or minus standard deviation. Comparisons were performed using the Student's $t$-test or single factor analysis of variance to test any statistically significant differences in the results between the experimental and control groups. Bonferroni correction was used where multiple comparisons were made. $P<0.05$ was considered statistically significant.

\section{Results}

\section{MACFI Expression in Melanoma Tissue in Mice}

Although abnormal MACF1 expression was found in numerous cancers, its expression in melanoma tissue remains largely unknown. In this study, we first determined the expression of MACF1 in B16F10 melanoma cells-induced tumors in mice. Figure 1A shows that MACF1 expression was significant higher in mouse melanoma tissue compared to that in adjacent 
A

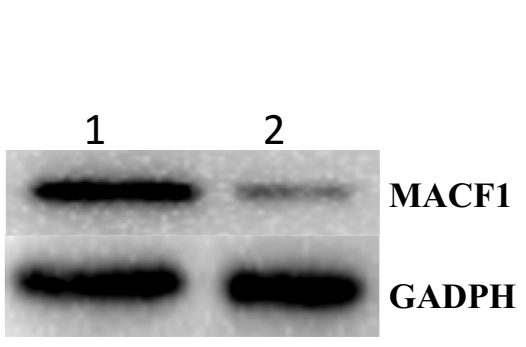

1. Mouse melanoma tissue

2. adjacent non-tumor tissues

C

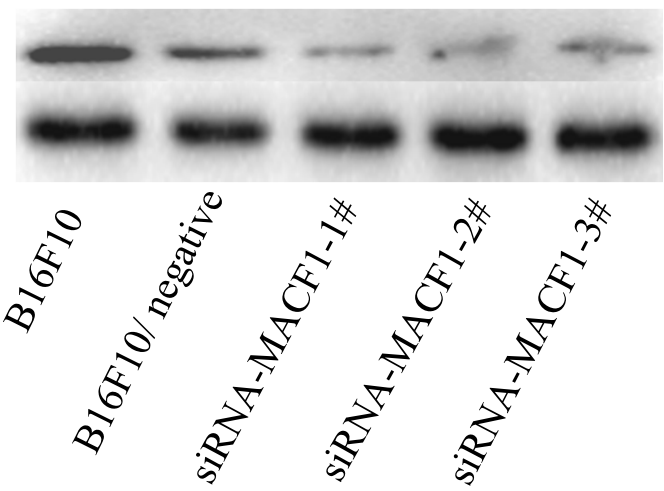

B
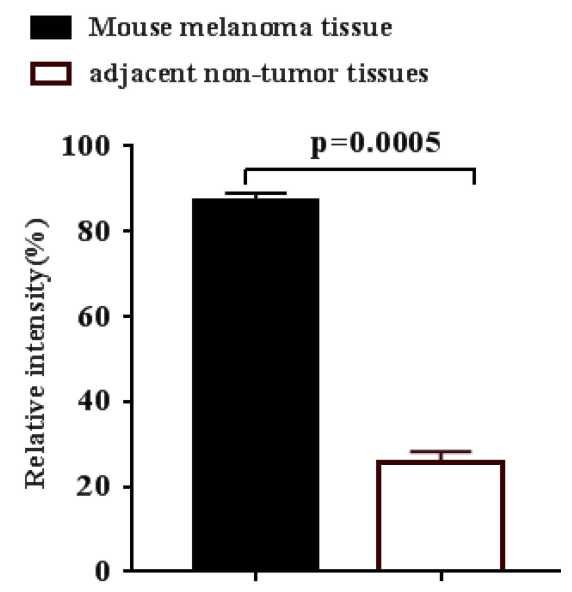

D

MACF1

GADPH

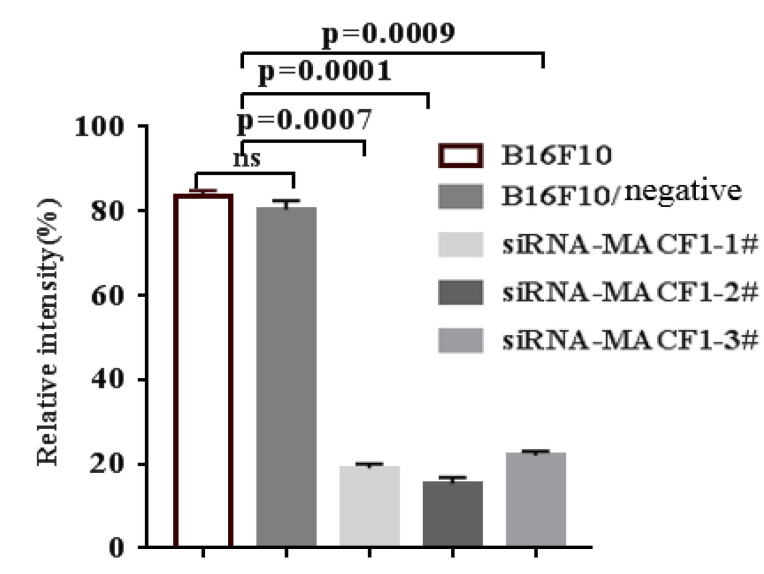

Figure I Western blot analysis of MACFI expression in different cells. (A) MACFI expression in BI6FI0 cell-induced tumor tissues and adjacent non-tumor tissues in mice. (B) Semi-quantification of MACFI expression referring to the statistically significant differences as indicated. (C) MACFI expression in BI6FI0 cells transfected with siRNAMACFI\#, siRNA-MACF2\#, and siRNA-MACF3\#, respectively. (D) Semi-quantification of MACFI expression in various cells.

non-tumor tissues as analyzed by Western blot analysis $(p<0.0005)$ (Figure 1B). Next, we used the constructed recombinants pSUPER-EGFP1- MACF1-siRNAs (siRNAMACF1\#, siRNA-MACF2\#, and siRNA-MACF3\#) that targeted the MACF1 encoding gene to transfect the B16F10 cells, respectively. Figure $1 \mathrm{C}$ shows that MACF1 expression was significantly decreased in B16F10/MACF1-RNAi cells compared with B16F10-negative cells, especially in siMACF2\#B16F10 cells (Figure 1D). The data indicated that construct siRNA-MACF2\# was appropriate for studying the effects of siRNA-MACF2\# (MACF1-RNAi, same below) on the behavior of B16F10 cells both in vitro and in vivo.

\section{Effects of Down-Regulating MACFI on Cellular Proliferation, Colony and Invasion of BI6FIO Cells}

To investigate the biological properties of B16F10-MACF1RNAi cells, we first conducted a cellular proliferation and colony forming assays to evaluate the ability of cell growth and amplification. Figure $2 \mathrm{~A}$ gives the proliferative activities dynamically every $24 \mathrm{~h}$ after B16F10/MACF1-RNAi and B16F10 cells had been seeded into 96-wells plate in complete medium. After $72 \mathrm{~h}$ of incubation, the OD value of B16F10 cells reached 1.6 \pm 0.21 , whereas B16F10/MACF1-RNAi cells were only $1.1 \pm 0.22$, which was statistically significant $(p<0.0255)$. Figure 2B shows that B16F10/MACF1-RNAi cells significantly decreased the colony forming rate compared with the B16F10 cells. The colony forming numbers were 6.02 \pm 0.81 for B16F10 cells and $5.94 \pm 1.42$ for B16F10-negative cells, whereas for B16F10/MACF1-RNAi cells, the colony forming numbers were $3.06 \pm 0.36$. Moreover, significant differences were observed between B16F10-MACF1-RNAi cells and B16F10 cells $(p<0.009)$, however no significant differences were observed between the B16F10/MACF1-RNAi cells and the B16F10-negative cells (Figure 2C).

Furthermore, the effect of knocking down MACF1 was evaluated on B16F10 cell invasive ability using a transwell 


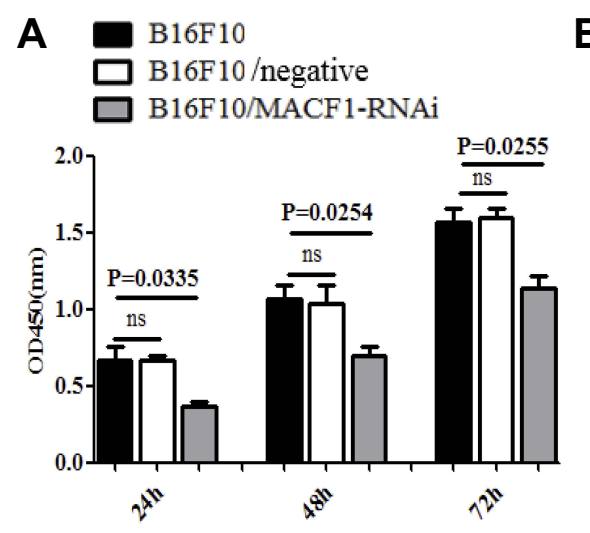

D

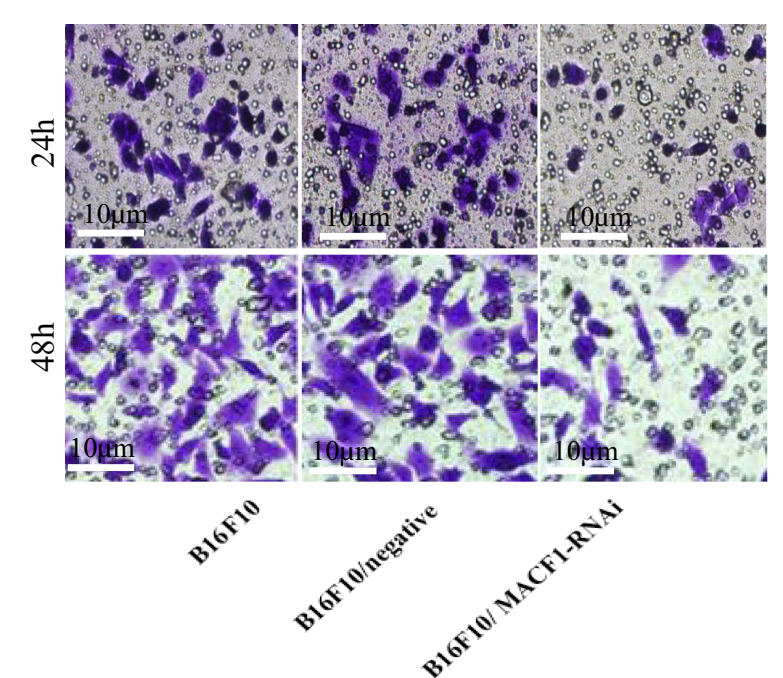

B C

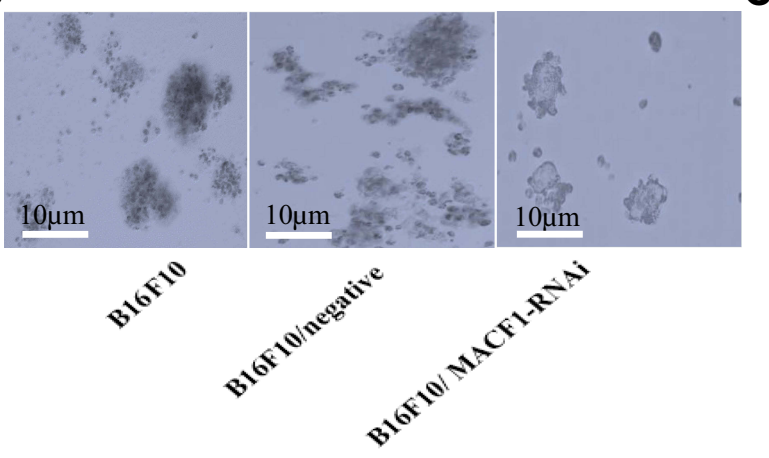

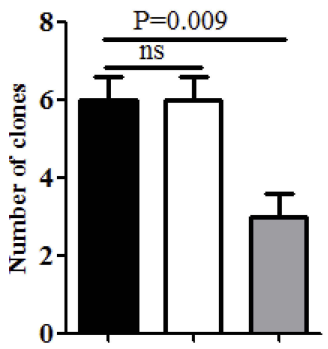

$\mathbf{F}$

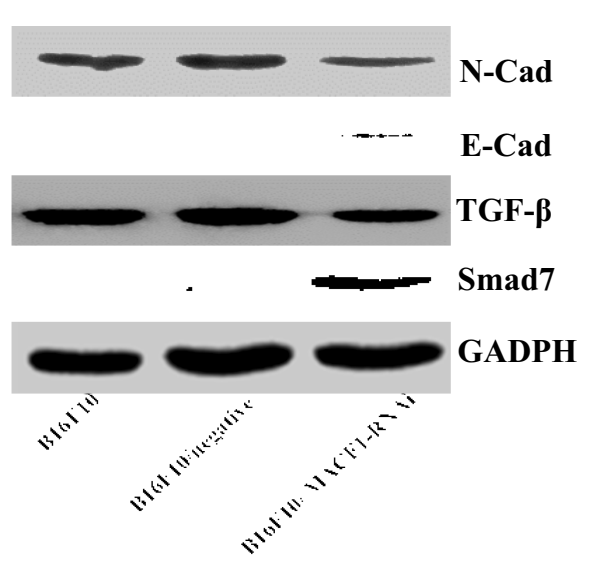

E

$\mathrm{B} 16 \mathrm{~F} 10$

$\mathrm{B} 16 \mathrm{~F} 10 /$ negative

B16F10/MACF1-RNAi

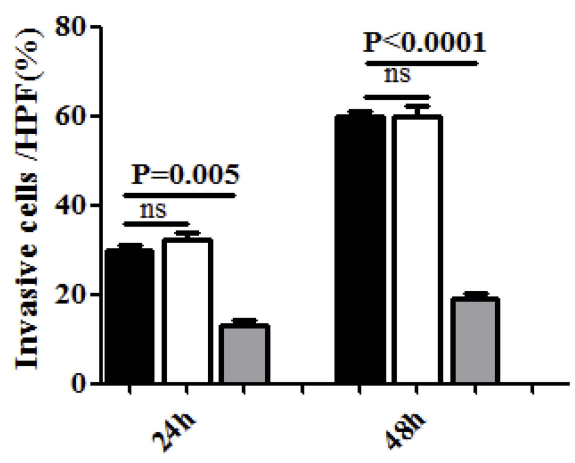

Figure 2 BI6FIO/MACFI-RNAi cells impact the ability of cellular proliferation, colony forming and invasion as well as EMT-related molecular expression in vitro. (A) The cell proliferative activity dynamically $24 \mathrm{~h}, 48 \mathrm{~h}$, and $72 \mathrm{~h}$ in the different cells. (B) Representative colony formation in the different cells. (C) Statistical analysis of the colony formation rate. (D) Invasive assay showing that the invasive ability of the BI6FIO/MACFI-RNAi cells was significantly decreased compared with that of BI6FI0 cells and BI6FI0-negative cells. (E) Statistical analysis of the cell invasive rate. (F) Western blot analysis showing the expression of N-cadherin, E-cadherin, TGF- $\beta$, and SMAD-7 in the different cells. (G) Semi-quantification of molecular expression, referring to the statistically significant differences as indicated. The labels of "BI6FI0-negative and BI6FI0/ MACFI-RNAi" denote BI6FIO cells transfected with scrambled shRNA and MACFI-RNAi.

invasive assay. Representative images of the cell invasive result are presented in Figure 2D. The invasive ability of B16F10/ MACF1-RNAi cells indicated a statistically significant decrease when compared to B16F10 cells (Figure 2E, $p<0.0005$ ) after culturing for $48 \mathrm{~h}$. Taken together, these results suggested that down-regulation of 
MACF1 in B16F10 cells clearly impacted its proliferation and colony formation and invasion abilities.

\section{Effects of Down-Regulating MACFI on the Expression of BI6FI0 Cell's \\ Mesenchymal and Epithelial Biomarkers}

To understand the mechanisms of the MACF1 knockdown on decreasing colony formation and invasion, we evaluated the expression of mesenchymal and epithelial biomarkers in B16F10/MACF1-RNAi cells. Increasing evidence has suggested that EMT is a process that is associated with many factors, in which N-Cadherin is a mesenchymal biomarker whereas E-Cadherin is an epithelial biomarker. Previous studies have shown that the TGF- $\beta$ pathway is often involved in EMT. However, regarding metastasis, Smad 7 can inhibit the TGF- $\beta$ canonical pathway through the inhibition of Smads $3 / 4$. Thus, TGF- $\beta$ and Smad 7 have opposite effects, interact with other proteins, and regulate TGF- $\beta$-independent signaling pathway contributing to the carcinogenic process $(22-24) .{ }^{21-23}$ In Figure 2E, Western blot analysis shows that the expression of TGF- $\beta$ and N-cadherin was significantly decreased ( $p<0.0001$ and $p<0.0017$, respectively), however the expression of E-cadherin and SMAD-7 was markedly increased in B16F10-MACF1-RNAi cells compared to B16F10 cells ( $p<0.0033$ and $p<0.0009$, respectively). However, no significant differences were observed between the B16F10 cells and the B16F10-negative cells (Figure 2F).

\section{MACFI Down-Regulation in BI6FIO \\ Cells Inhibits Tumor Growth and Metastasis in Mouse Model}

After evaluating the effects of down-regulated MACF1 expression on B16F10 cell's colony formation and invasion in vitro, we evaluated if these effects would influence the tumorigenicity and metastatic potential of the B16F10/ MACF1-RNAi cells in vivo. Figure 3A shows representative

\section{A}

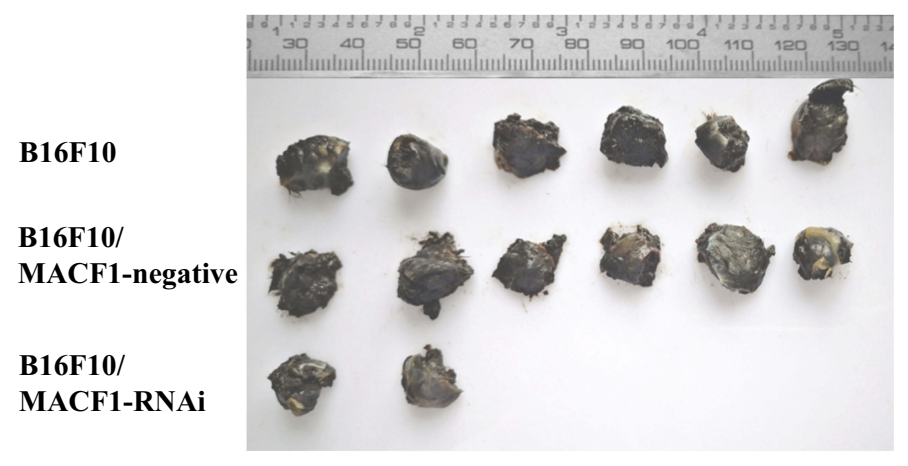

C

$\mathrm{B} 16 \mathrm{~F} 10$

B16F $10 /$ negative

$\square$ B16F10/MACF1-RNAi

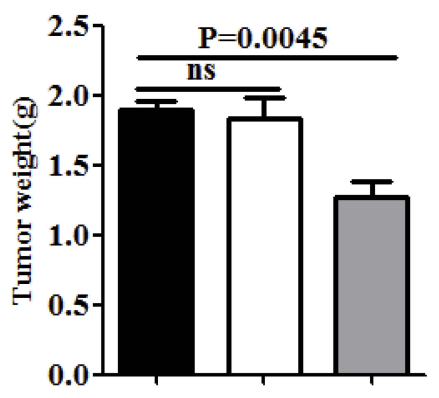

D
B
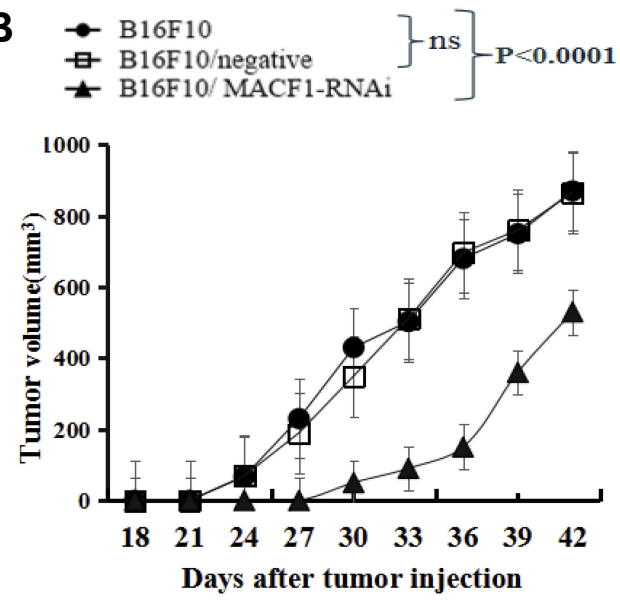

E
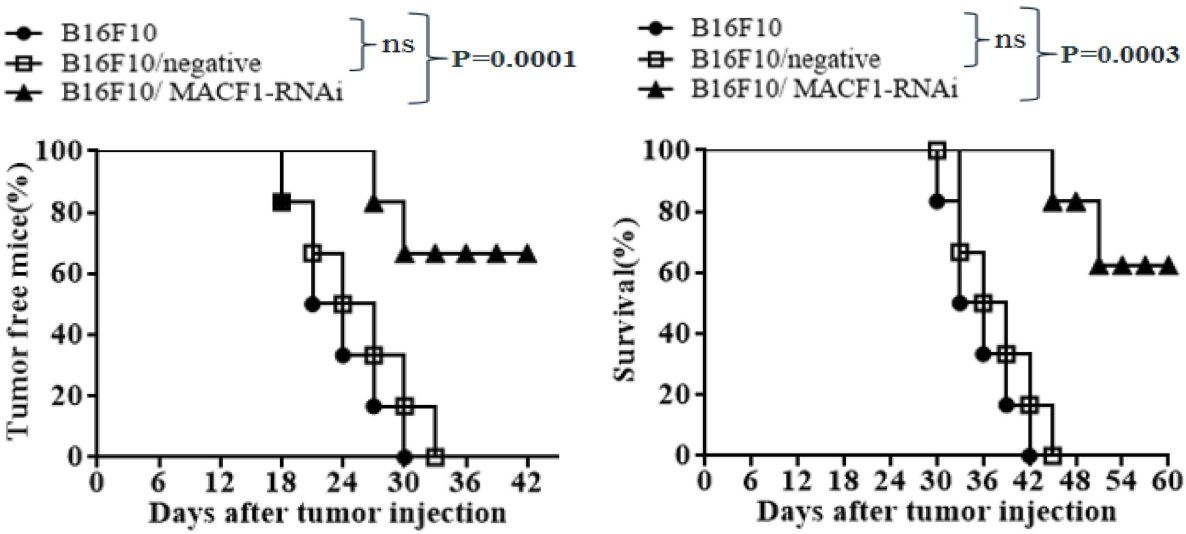

Figure 3 Effects of the down-regulation of MACFI on tumor-bearing mouse growth and survival. (A) Tumor sizes of tumors that were removed from BI6FI0 cell-induced tumor mice injected with BI6FI0/MACFI-RNAi, BI6FI0-negative, and BI6FI0 cells, respectively. (B) Changes in the dynamic state of tumor growth. (C) The weight of the mass tumors. (D) Tumor-free mice injected with the three types of cells. (E) Survival of tumor-bearing mice injected with the different cells. 
images of the tumor sizes and demonstrates that in mice injected with $2 \times 10^{5}$ B16F10 cells, one mouse developed a visible tumor on Day 18, two mice on Day 21, and one mouse on Day 24, Day 27, and Day 30, respectively. Moreover, in the $2 \times 10^{5} \mathrm{~B} 16 \mathrm{~F} 10$-negative cell group, one mouse developed a visible tumor on Day 18, and one mouse on Day 21, on Day 24, Day 27, on Day 30, and Day 33, respectively. In contrast, only 2 of the 6 mice injected with $2 \times 10^{5}$ B16F10- MACF1-RNAi cells developed tumors on Day 27 and Day 30, whereas the remaining 4 mice did not develop any tumors throughout the 42-day observation period. The dynamic state change of tumor growth, tumor weight, and the percentages of tumor-free mice are presented in Figure 3B-D. Figure 3E shows the result of mouse survival and quantity. The data showed that when compared with mice injected with B16F10 cells, tumor growth was significantly reduced both in size and latency time in mice injected with B16F10/MACF1-RNAi cells (Figure 3B, $* * * p<0.0009$ ).

In addition, it was found that a large, visible metastatic foci in the lungs were increased in mice that were intravenously injected with $2 \times 10^{5}$ B16F10 cells when compared to mice that were injected intravenously with $2 \times 10^{5}$ B16F10/MACF1- RNAi cells (Figure 4A). The visible metastatic foci were more abundant in B16F10 cells and B16F10- negative cells (Figure 4B) when compared to B16F10/MACF1-RNAi cells (Figure 4C), which was a statistical significance as shown in Figure 4D.

To evaluate the underlying molecular mechanisms of down-regulating $M A C F 1$ gene expression, we detected the expression of TGF- $\beta, \mathrm{N}$-cadherin, E-cadherin and SMAD-7 in tumor tissues of mice challenged with the different treated B16F10 cells. Figure 5A depicts the expression of TGF- $\beta$, N-cadherin, E-cadherin, and SMAD-7 in tumor tissue as analyzed by Western blot analysis. Consistent with the data obtained by Western blot analysis in the different B16F10 cells (Figure 2E), the expression of E-cadherin and SMAD-7 were higher in tumor tissues derived from mice challenged with B16F10/MACF1-RNAi cells when compared to tumor tissues in B16F10 cell-challenged mice. However, the expression of TGF- $\beta$ and N-cadherin were lower when compared to tumor tissues of mice challenged by the B16F10 cells. The difference between the B16F10/ MACF1- RNAi cells and the B16F10 cells was statistically significant (Figure 5B).

The immunohistochemistry results agreed with results of Western blot analysis, and showed that a reduction in TGF- $\beta$ and N-cadherin expression but an increase in the expression of E-cadherin and SMAD-7 in B16F10/ MACF1-RNAi cell driven melanoma tissues was observed (Figure 6A), and the differences were statistically significant (Figure 6B). These results showed that knockdown of MACF1 expression inhibited B16F10 cell tumorigenicity and B16F10 melanoma distant metastasis in mouse lungs, thereby suggesting that B16F10 cell's EMT was inhibited through a molecular association of the inhibition of TGF- $\beta$ and $\mathrm{N}$-cadherin and the increase in the E-cadherin and SMAD-7 expression in tumor tissues.
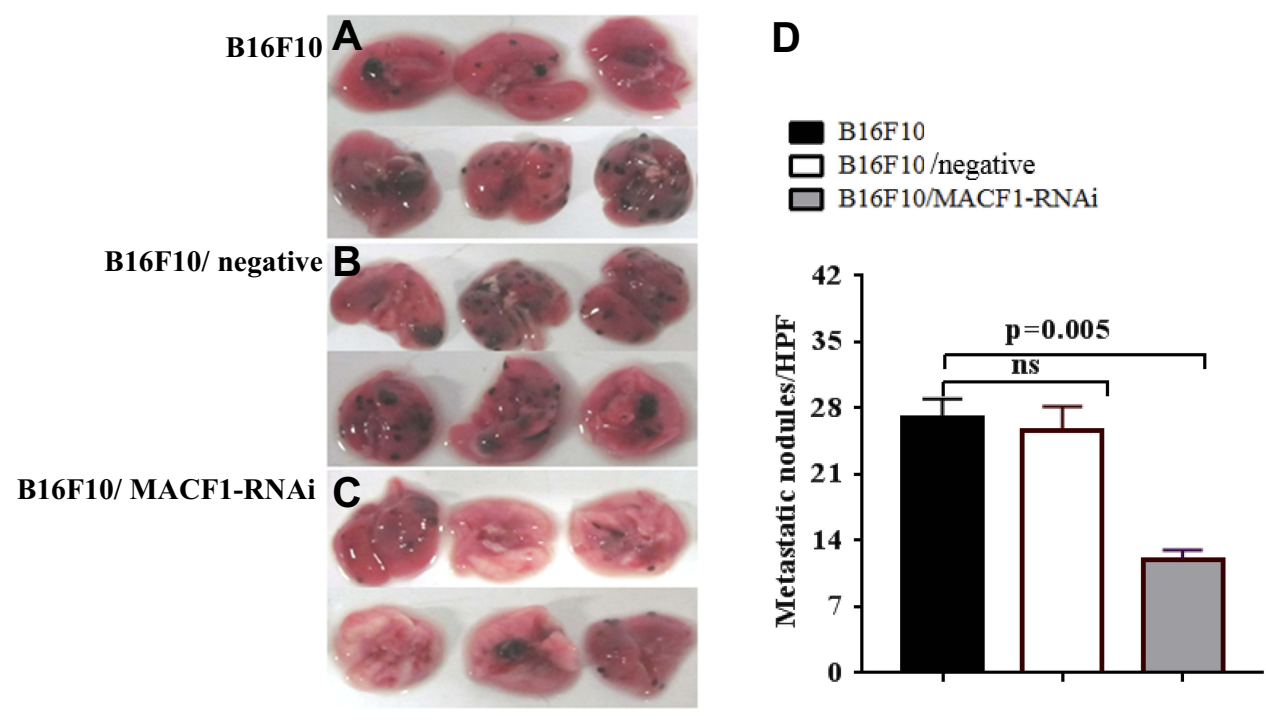

Figure 4 Effects of the down-regulation of MACFI in BI6FIO cells on tumor lung metastasis. (A, B, C) Representative images of lung metastasis in mice that were intravenously injected with the different BI6FI0 cells 30 days after injection. (D) Statistical analysis of lung metastatic tumor node count in BI6FI0/MACFI-RNAi, BI6FI0negative, and BI6FIO cell groups, referring to statistically significant differences as indicated by $P$ values. 
A

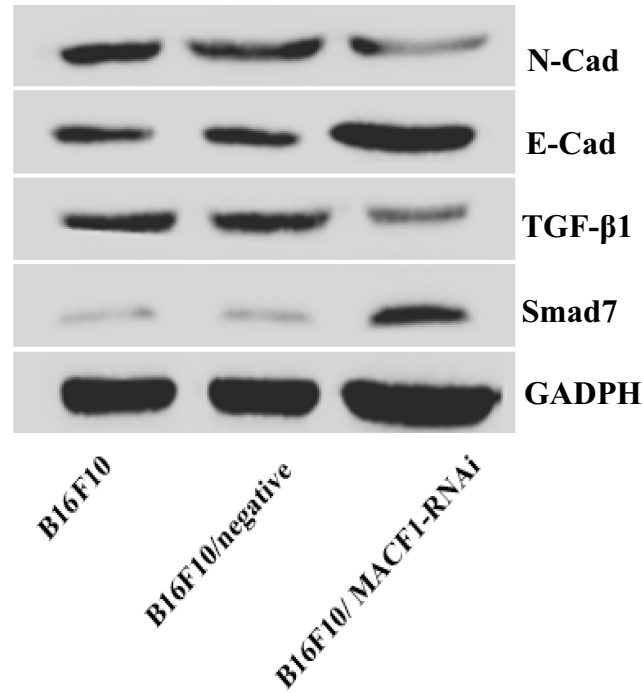

B
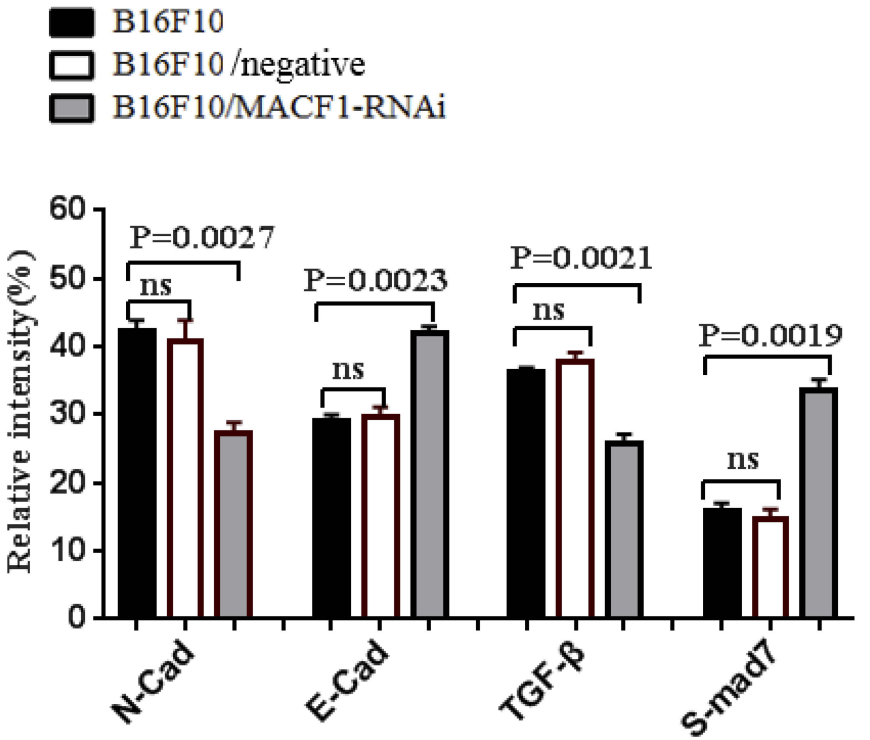

Figure 5 EMT-related molecular expression detected by Western blot assay. (A) Representative images of bands showing the molecular expression of N-cadherin, E-cadherin, TGF- $\beta$, and SMAD-7 in tumor tissues derived from BI6FI0 cell-induced tumor mice 42 days after mice were challenged with the different BI6FI0 cells. (B) Semiquantification of molecular expression, referring to the statistically significant differences as indicated.

\section{Discussion}

Although most new cases of melanoma are thin (no metastases), a significant percentage of patients still experiences disease progression, and metastases are responsible for the majority patient mortality. Therefore, recent studies have focused on the molecular mechanisms underlying melanoma progression and metastases as well as on novel strategies for early effective therapy, including immune checkpoint inhibitors. ${ }^{24-26}$ However, how to successfully inhibit melanoma metastases remains a major barrier to be overcome. Recently, increasing evidence has revealed that MACF1 plays an essential role in various cellular processes, and is involved in the control of microtubule dynamics that relates with cell proliferation and migration, thereby suggesting that MACF1 function is an important for cellular motility. ${ }^{7,27,28}$ In previous studies, it had been shown that MACF1 was expressed in a different tumors, ${ }^{10,29}$ however its impact on melanoma metastases is unknown. In the present study, we aimed to corroborate the function of MACF1 by using RNA interference to downregulate MACF expression in B16F10 murine melanoma cells and to further evaluate the metastatic potential of B16F10/MACF1-RNAi cells both in vitro and in vivo.

After having demonstrated the abnormal expression of MACF1 in B16F10 cells-induced tumors in mice (Figure 1), we investigated the influence of down-regulated MACF1 expression in B16F10 cells on cellular colony formation and invasion, which are an essential potential for malignant tumor invasion and metastasis. ${ }^{14,15}$ Our data demonstrated that the cellular proliferation and colony formation numbers in the B16F10/MACF1-RNAi cells were markedly decreased. In addition, the invasion ability was notablely reduced in contrast with B16F10 cells (Figure 2), which suggested that down-regulation of MACF1 expression attenuated the B16F10 cell's clonogenic ability and metastatic potential in vitro.

The previous studies suggested that the colony formation in vitro associated positively with the in vivo stage of cancer progress. $^{30-32}$ Therefore, any decrease in the tumor cellular colony formation ability in vitro may lead to reduced tumorigenesis in the in vivo mouse model. ${ }^{14}$ To this end, we further evaluated the impact of MACF1 down-regulation on the tumorigenesis of B16F10 cells in mice. As predicted, we found that the latest tumor formation, the smallest tumor sizes, and the longest survival of mice were observed in B16F10/MACF1-RNAi cells-challenged mice when compared to B16F10 or B16F10-negative cells- challenged mice, which demonstrated the notable inhibition of B16F10/ MACF1-RNAi cell's tumorigenesis. Moreover, a visible metastatic focus in the lungs was significantly reduced in mice that were intravenously injected with B16F10/MACF1RNAi cells when compared with control mice (Figure 5). 
To fully understand the antimetastatic molecular mechanisms, we tested the EMT-related molecular expression, including a mesenchymal biomarker and an epithelial biomarker in B16F10 cells-induced tumor tissues. Western blot analysis from in vivo animal experiments were consistent with in vitro cellular experiments, indicating an increase in expression of E-cadherin and SMAD-7 and a decrease in expression of TGF- $\beta$ and $\mathrm{N}$-cadherin in tumor tissues in the mice challenged with B16F10/MACF1-RNAi cells when compared to mice challenged with B16F10 cells or B16F10negative cells. Furthermore, these results were supported by immunohistochemical analysis, which were based on tumor sections in which high expression of E-cadherin (epithelial cell phenotype) and SMAD-7, and low expression of $\mathrm{N}$-cadherin (mesenchymal cell phenotype) and TGF- $\beta$ were demonstrated, respectively (Figure 6).

It is known that malignant melanoma possesses epitheliallike characteristics through interactions between cancer cells and their microenvironment to develop phenotype switching in spite of melanoma originated from non-epithelial cells, ${ }^{33-35}$ and that the loss of E-cadherin function or expression is implicated in cancer progression and metastasis, and Vimentin is often used as a marker for mesenchymally-derived cells or cells undergoing the EMT during both normal development and tumor metastatic progression. ${ }^{13,21}$ Therefore, reduced expression of E-cadherin or Smad-7 function resulted in melanoma progression and metastasis, ${ }^{36}$ whereas an increase in $\mathrm{N}$-cadherin and TGF- $\beta$ expression was closely related with B16F10 melanoma cell's EMT. This may be because the TGF$\beta 1$ signal by membrane-bound heteromeric serine- threonine kinase receptor complexes leads to the phosphorylation of proteins of the Smad family. Phosphorylated Smad2/Smad3 accumulates in the nucleus and could serve as a transcription factor. ${ }^{37,38}$ We hypothesized that the increase in E-cadherin and reduction in $\mathrm{N}$-cadherin expression in B16F10 cells-induced tumor tissue may lead to an increase in Smad-7 expression. However, a reduction in TGF- $\beta 1$ expression reduced phosphorylation of Smad2 and Smad3, which resulted in inhibition of B16F10/MACF1-RNAi cell's EMT.

Our findings were in line with the data presented in a recent report that showed that inhibition of MACF1 expression selectively suppressed glioblastoma cell proliferation and migration in cell lines established from patient derived xenograft mouse models. ${ }^{10}$ Therefore, recent studies including ours

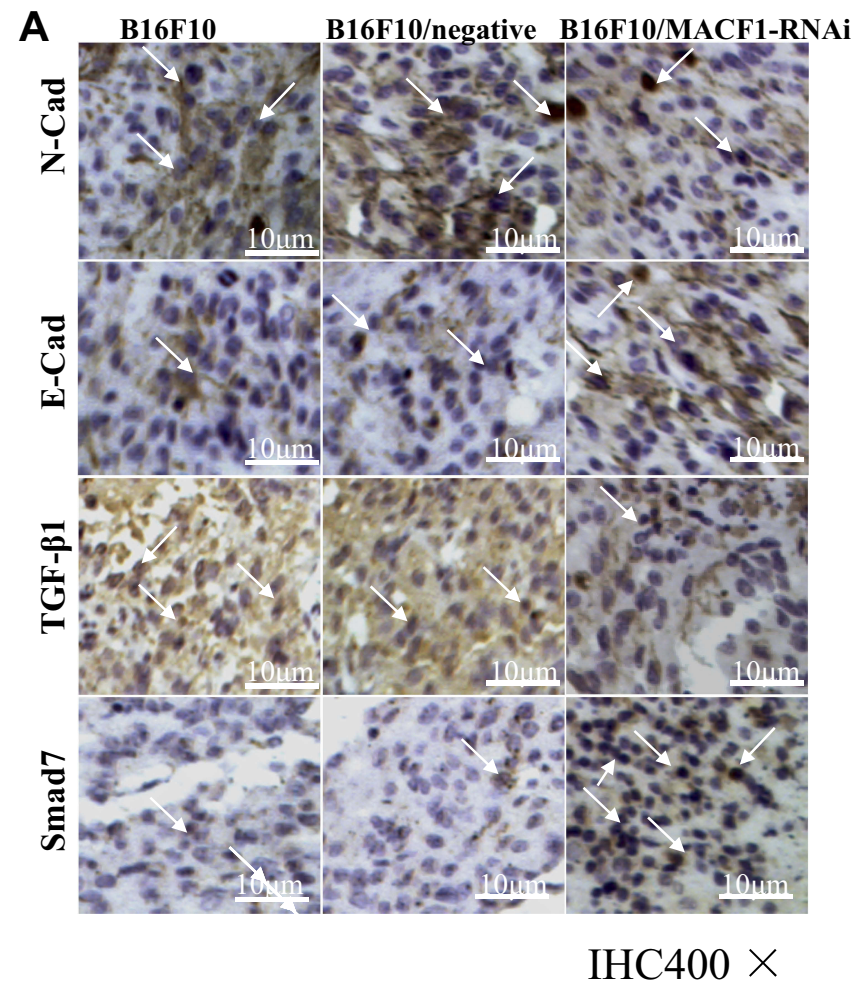

B
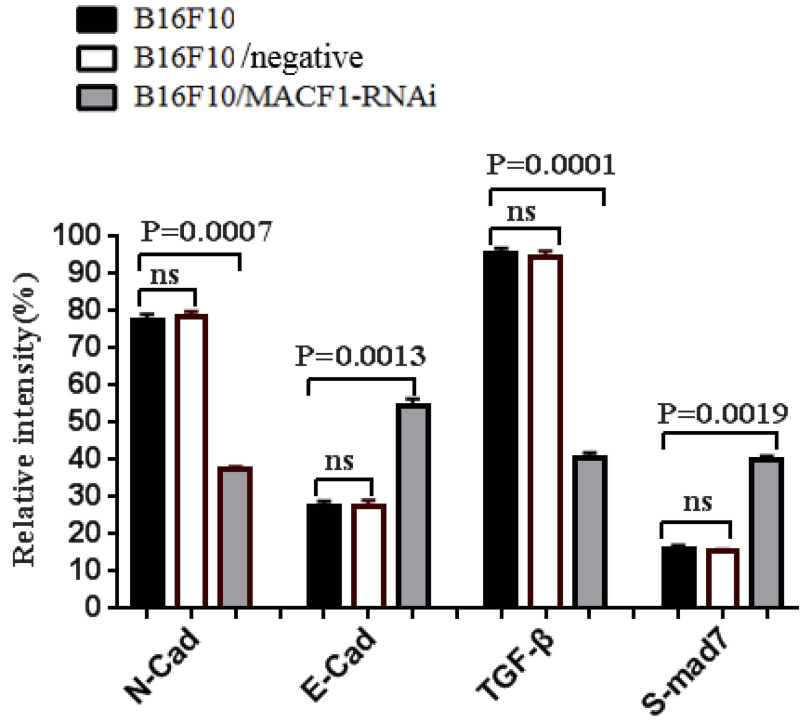

Figure 6 Analysis of EMT associated molecular expression in BI6FIO cell-induced tumor tissues. (A) Immunohistochemical analysis showing representative images of expression of N-cadherin, E-cadherin, TGF- $\beta$, and SMAD-7 in tumor cells (400x). Brown cells represent N-cadherin, E-cadherin, TGF- $\beta$, and SMAD-7 positive cells (pointed to by white arrows), respectively, in tumor tissues. (B) Semi-quantification of molecular expression, referring to the statistically significant differences as indicated. Scale bars: $10 \mu \mathrm{m}$. 
have provided evidence to confirm that MACF1 plays a role in the EMT process in metastatic cancers.

Although the above-mentioned findings are promising, a limitations of the present study is that molecular linkages that cause MACF1 to impact the changes in expression of E-cadherin and $\mathrm{N}$-cadherin are unknown, and that the analysis of matrix metalloproteinase- 9 expression level is lacked, which plays important roles in the progression of various types of tumors. ${ }^{39}$ In addition, the lack of E-cad and N-cad knockdown is another limitation in the present study. Further studies that focus on the mechanism of inhibiting the EMT program mediated by down-regulating MACF1 in B16F10 cells remains to be investigated.

In conclusion, our data showed that B16F10/MACF1RNAi cells decreased its colony formation, invasion, metastasis, and tumorigenicity via inhibiting the EMT program. Our findings supports the hypothesis that an enhancing melanoma therapeutic efficacy may be acquired by inhibiting MACF1 expression, a therapeutic target for melanoma treatment.

\section{Ethics Approval}

The Animal Research Ethics Board of Jiangnan University approved all experimental protocols.

\section{Abbreviations}

MACF1, microtubule actin cross-linking factor 1; CTLA-4, cytotoxic T-lymphocyte antigen-4; PD-1/PDL1, programmed death 1; EMT, epithelial to mesenchymal transition.

\section{Data Sharing Statement}

All data analyzed and generated in this research report are enclosed in the article.

\section{Acknowledgments}

The authors wish to acknowledge Professor Pingsheng Chen (Department of Pathophysiology, School of Medicine, Southeast University, Nanjing, China) for the generous gift of the B16F10 cell line.

\section{Author Contributions}

$\mathrm{XYW}, \mathrm{XJ}$, and ZCW conducted the experiments, XYW, FSZ, and JD analyzed the data, XYW, JD, and FSZ wrote the manuscript, and JD revised the manuscript. All authors reviewed and JD final approved the version to be published. All authors contributed to data analysis, drafting or revising the article, gave final approval of the version to be published and agree to be accountable for all aspects of the work in ensuring that questions related to the accuracy or integrity of any part of the work are appropriately investigated and resolved.

\section{Funding}

This work was supported by the national first-class discipline program of Food Science and Technology, Jiangnan University (JUFSTR20180101, Wuxi, China), and in part by the Medical Talented Youth in Engineering of Science and Education for Public Health, Wuxi City, Wuxi China (QNRC058).

\section{Disclosure}

The authors declared no potential conflicts of interest with respect to research, authorship, and/or publication of this manuscript.

\section{References}

1. Dantonio PM, Klein MO, Freire MRVB, et al. Exploring major signaling cascades in melanomagenesis: a rationale route for targeted skin cancer therapy. Biosci Rep. 2018;38:BSR20180511. doi:10. 1042/BSR20180511

2. Somasundaram R, Herlyn M. Nivolumab in combination with ipilimumab for the treatment of melanoma. Expert Rev Anticancer Ther. 2015;15(10):1135-1141. doi:10.1586/14737140.2015.1093418

3. Villareal MO, Sato Y, Matsuyama K, et al. Daphnane diterpenes inhibit the metastatic potential of B16F10 murine melanoma cells in vitro and in vivo. BMC Cancer. 2018;18(1):856. doi:10.1186/ s12885-018-4693-y

4. Zhao Y, Schaafsma E, Gorlov IP, et al. A leukocyte infiltration score defined by a gene signature predicts melanoma patient prognosis. $\mathrm{Mol}$ Cancer Res. 2019;17(1):109-119. doi:10.1158/1541-7786

5. Garcia CA, El-Ali A, Rath TJ, et al. Neurologic immune-related adverse events associated with adjuvant ipilimumab: report of two cases. J Immunother Cancer. 2018;6(1):83. doi:10.1186/s40425-0180393-z

6. Wang X, Zhao F, Shi F, et al. Reinforcing B16F10/GPI-IL-21 vaccine efficacy against melanoma by injecting mice with shZEB1 plasmid or miR200c agomir. Biomed Pharmacother. 2016;80:136-144. doi:10. 1016/j.biopha.2016.03.013

7. Goryunov D1, Liem RK. Microtubule-actin cross-linking factor 1: domains, interaction partners, and tissue-specific functions. Methods Enzymol. 2016;569:331-353. doi:10.1016/bs.mie.2015.05.022

8. Bouameur JE, Favre B, Borradori L. Plakins, a versatile family of cytolinkers: roles in skin integrity and in human diseases. $J$ Invest Dermatol. 2014;134(4):885-894. doi:10.1038/jid.2013.498

9. Miao Z, Ali A, Hu L, et al. Microtubule actin cross-linking factor 1, a novel potential target in cancer. Cancer Sci. 2017;108 (10):1953-1958. doi:10.1111/cas.13344

10. Afghani N, Mehta T, Wang J, et al. Microtubule actin cross-linking factor 1, a novel target in glioblastoma. Int $J$ Oncol. 2017;50 (1):310-316. doi:10.3892/ijo.2016.3798

11. Dou J, Liu P, Zhang X. Cellular response to gene expression profiles of different hepatitis $\mathrm{C}$ virus core protein in Huh-7 cell line with microarray analysis. J Nanosci Nanotechnol. 2005;5(8):1230-1235. doi:10.1166/jnn.2005.209 
12. Dou J, Wang Y, Yu F, et al. Protection against Mycobacterium tuberculosis challenge in mice by DNA vaccine Ag85AESAT-6-IL-21 priming and BCG boosting. Int $J$ Immunogenet. 2012;39(2):183-190. doi:10.1111/j.1744-313X.2011.01066.x

13. Chen D, Wang J, Zhang Y, et al. Effect of down-regulated transcriptional repressor ZEB1 on the epithelial-mesenchymal transition of ovarian cancer cells. Int J Gynecol Cancer. 2013;23(8):1357-1366. doi:10.1097/IGC.0b013e3182a5e760

14. Dou J, Li Y, Zhao F, et al. Identification of tumor stem like cells in mouse myeloma cell lines. Cell Mol Biol (Noisy-Le-Grand). 2009;55 (Suppl):OL1151-60.

15. Zhang H, Cai K, Wang J, et al. MiR-7, inhibited indirectly by lincRNA HOTAIR, directly inhibits SETDB1 and reverses the EMT of breast cancer stem cells by down-regulating the STAT3 pathway. Stem Cells. 2014;32(11):2858-2868. doi:10.1002/stem.1795

16. Ngora H, Galli UM, Miyazaki K, et al. Membrane-bound and exosomal metastasis- associated $\mathrm{C} 4.4 \mathrm{~A}$ promotes migration by associating with the $\alpha(6) \beta(4)$ integrin and MT1-MMP. Neoplasia. 2012;14 (2):95-107. doi:10.1593/neo.111450

17. He X, Wang J, Zhao F, et al. Antitumor efficacy of viable tumor vaccine modified by heterogenetic ESAT- 6 antigenand cytokine IL-21 in melanomatous mouse. Immunol Res. 2012;52(3):240-249. doi:10.1007/s12026-012-8332-4

18. Wang X, He X, Zhao F, et al. Regulation gene expression of miR200c and ZEB1 positively enhances effect of tumor vaccine B16F10/ GPI-IL-21 on inhibition of melanoma growth and metastasis. J Transl Med. 2014;12:68. doi:10.1186/1479-5876-12-68

19. Zhao F, He X, Wang Y, et al. Decrease of ZEB1 expression inhibits the B16F10 cancer stem-like properties. Biosci Trends. 2015;9 (5):325-334. doi:10.5582/bst.2015.01106

20. Hu W, Wang J, Dou J, et al. Augmenting therapy of ovarian cancer efficacy by secreting IL-21 human umbilical cordblood stem cells in nude mice. Cell Transplant. 2011;20(5):669-680. doi:10.3727/ 096368910X536509

21. Burk U, Schubert J, Wellner U, et al. A reciprocal repression between ZEB1 and members of the miR-200 family promotes EMT and invasion in cancer cells. EMBO Rep. 2008;9(6):582-589. doi:10.1038/ embor.2008.74

22. Zhang P, Wang L, Rodriguez-Aguayo C, et al. miR-205 acts as a tumour radiosensitizer by targeting ZEB1 andUbc13. Nat Commun. 2014;5:5671. doi:10.1038/ncomms6671

23. Stolfi C, Marafini I, De Simone V, et al. The dual role of Smad7 in the control of cancer growth and metastasis. Int J Mol Sci. 2013;14 (12):23774-23790. doi:10.3390/ijms141223774

24. Reiter JG, Makohon-Moore AP, Gerold JM, et al. Minimal functional driver gene heterogeneity among untreated metastases. Science. 2018;361(6406):1033-1037. doi:10.1126/science.aat7171

25. Tas F, Erturk K. Palpebral cutaneous melanomas: a review of 17 cases from a tertiary center. Int J Dermatol. 2019;58:75-79. doi:10.1111/ijd.14212
26. Wieland A, Kamphorst AO, Adsay NV, et al. T cell receptor sequencing of activated CD8 $\mathrm{T}$ cells in the blood identifies tumor-infiltrating clones that expand after PD-1 therapy and radiation in a melanoma patient. Cancer Immunol Immunother. 2018;67(11):1767-1776. doi:10.1007/s00262-018-2228-7

27. Jørgensen LH, Mosbech MB, Færgeman NJ, et al. Duplication in the microtubule- actin cross-linking factor 1 gene causes a novel neuromuscular condition. Sci Rep. 2014;4:5180. doi:10.1038/srep05180

28. Chen HJ, Lin CM, Lin CS, et al. The role of microtubule actin crosslinking factor 1 (MACF1) in the Wnt signaling pathway. Genes Dev. 2006;20(14):1933-1945. doi:10.1101/gad.1411206

29. Ma Y, Zhang P, Wang F, et al. An integrated proteomics and metabolomics approach for defining oncofetal biomarkersin the colorectal cancer. Ann Surg. 2012r;255(4):720-730. doi:10.1097/SLA.0b013e $31824 a 9 a 8 b$

30. Matsui W, Huff CA, Wang Q, et al. Characterization of clonogenic multiple myeloma cells. Blood. 2004;103(6):2332-2336. doi:10.1 182/blood-2003-09-3064

31. Ouyang L, Shen LY, Li T, et al. Inhibition effect of Oncostatin M on metastatic human lung cancer cells $95 \mathrm{D}$ in vitro and on murine melanoma cells B16BL6 in vivo. Biomed Res. 2006;27(2):197-202. doi:10.2220/biomedres.27.197

32. Dou J, Wen P, Hu W, et al. Identifying tumor stem-like cells in mouse melanoma cell lines by analyzing the characteristics of side population cells. Cell Biol Int. 2009;33(8):807-815. doi:10.1016/j.cellbi. 2009.05.003

33. Rangel MC, Karasawa H, Castro NP, et al. Role of Cripto-1 during epithelial-to- mesenchymal transition in development and cancer. $\mathrm{Am}$ J Pathol. 2012;180(6):2188-2200. doi:10.1016/j.ajpath.2012.02.031

34. Kemper K, de Goeje PL, Peeper DS, et al. Phenotype switching: tumor cell plasticity as aresistance mechanism and target for therapy. Cancer Res. 2014;74(21):5937-5941. doi:10.1158/0008-5472

35. Lu L, Tao H, Chang AE, et al. Cancer stem cell vaccine inhibits metastases of primary tumors and induces humoral immune responses against cancer stem cells. Oncoimmunology. 2015;4(3): e990767. doi:10.4161/2162402X.2014.990767

36. Balch C, Nephew KP. The role of chromatin, microRNAs, and tumor stem cells in ovarian cancer. Cancer Biomark. 2010-2011;8(4-5):203-221. doi:10.3233/CBM-2011-0214

37. Javelaud D, Alexaki VI, Dennler S, et al. The TGF- $\beta /$ SMAD/GLI2 signaling axis in cancer progression and Metastasis. Cancer Res. 2011;71(17):5606-5610. doi:10.1158/0008-5472.CAN-11-1194

38. Ulloa L, Doody J, Massagué J. Inhibition of transforming growth factor-beta/SMAD signalling by the interferon-gamma/STAT pathway. Nature. 1999;397(6721):710-713. doi:10.1038/17826

39. Moawed FS, El-Sonbaty SM, Mansour SZ. Gallium nanoparticles along with low-dose gamma radiation modulate TGF- $\beta$ /MMP-9 expression in hepatocellular carcinogenesis in rats. Tumour Biol. 2019;41(3):1010428319834856. doi:10.1177/1010428319834856
Cancer Management and Research is an international, peer-reviewed open access journal focusing on cancer research and the optimal use of preventative and integrated treatment interventions to achieve improved outcomes, enhanced survival and quality of life for the cancer patient.
The manuscript management system is completely online and includes a very quick and fair peer-review system, which is all easy to use Visit http://www.dovepress.com/testimonials.php to read real quotes from published authors. 